\title{
Concept, Simulation Studies and Design for a Novel Concept of the Hydraulic Binary Counter
}

\author{
Rudolf Scheidl and Matthias Scherrer \\ Institute of Machine Design and Hydraulic Drives, Johannes Kepler University Linz, Linz, Austria \\ E-mail: Rudolf.scheidl@jku.at, Matthias.scherrer@jku.at
}

\begin{abstract}
For the realization of compact and lightweight digital hydraulic cylinder drives for exoskeleton actuation the hydraulic binary counter concept was proposed and studied previously. This counter principle is based on hydraulically piloted switching valves which feature a hysteretic response with respect to the pilot pressure. In first prototypes of that counter bistable mechanical buckling beams realized the hysteretic response. Their performance suffered from high friction in the hinges and high local stresses. Furthermore, they require tight manufacturing tolerances not only of themselves but also of their bearing structure. In this paper, hydraulic feedback from the multi-chamber cylinders is proposed and investigated to realize the reset of lower order valves when a higher order valve switches. To make the hydraulic feedback independent from the system pressure feedback to the valve is done via spring compression. This principle makes bi-stable elements obsolete. The functioning of this principle for a small drive for exoskeleton use is proven by a simple mathematical model and its numerical solution by a MATLAB program. An exemplary embodiment design of the valves with the proposed feedback mechanism shows the feasibility of its practical realization. The application of this concept is not limited to small drives but can be applied for larger drives where multi-chamber cylinders are advantageous, such as for excavators, to save cost and installation space of the many solenoid valves required otherwise.
\end{abstract}

Keywords: Multi chamber cylinder, digital fluid power, hydraulic binary counter

\section{Introduction}

The use of a hydraulic counter principle for the control of a multi-chamber cylinder drive was first studied 2011 in [1] for the realization of a digital pendant of the analog linear hydraulic amplifier. This device transfers a low force mechanical input motion $x_{d}$ into a proportionate output motion $x_{a}$ against a high force $F$; see Figure 1. $x_{d}$ is input mechanically to the spool, $x_{a}$ to the housing of a proportional valve which leads to pilot flows $Q_{X I}$ and $Q_{X P}$ into or out of the pilot line. The pilot flow and the resulting pilot pressure $p_{C}$ make the four $3-2$ way valves switching on or off in a consecutive, binary manner such that the number values of the binary states follow the natural number series to raise or lower the resultant force according to the cylinder pressures and cylinder areas. $Q_{X I}$ leads to an integral behavior of the system, because it provides a stepping up or down of the resultant hydraulic force as long as $x_{d}$ is larger or smaller than $x_{a}$. The proportional part of this feedback control mechanism given by $Q_{X P}$ stabilizes the response behavior. Later, this concept was taken up to realize a hydraulic piloting actuation of multi-chamber cylinder valves for exoskeleton use to save weight of the relatively heavy solenoids $[2,3]$. The counting principle studied in these papers was based on a hysteresis response of the four 32 way switching valves for the four cylinder chambers. This response manages the reset of all lower order bits when a certain bit performs the counting step. The snap through property of a buckling beam was selected as a passive mechanical realization but turned out to suffer from a too high friction in the joints [4]. Furthermore, the concept imposes narrow design rules for the valves of the different binary stages and tight tolerances, which 
means an additional complexity for system design. The use of repulsive permanent magnets [5], a passive principle too, leads to a relatively complex and bulky design.

The binary counter principle can not only save weight and space but also cost of the solenoid valves, which is a high burden for the economically feasible realization of multi-chamber cylinder drives. For instance, cost is a major design criterion of small and mid-size range mobile working machines, for which these drives are seen a promising way for substantial fuel savings [7-9]. For the valve sizes required fort such mobile machinery applications passive concepts of binary counting are not feasible because of the high flow and friction forces. Such valves may use an internal hydraulic feedback by additional metering edges, as already suggested in [1] and sketched in the left side drawing of Figure 2. However, those edges would cause difficulties for very small valves, as needed for hydraulic exoskeleton actuation.

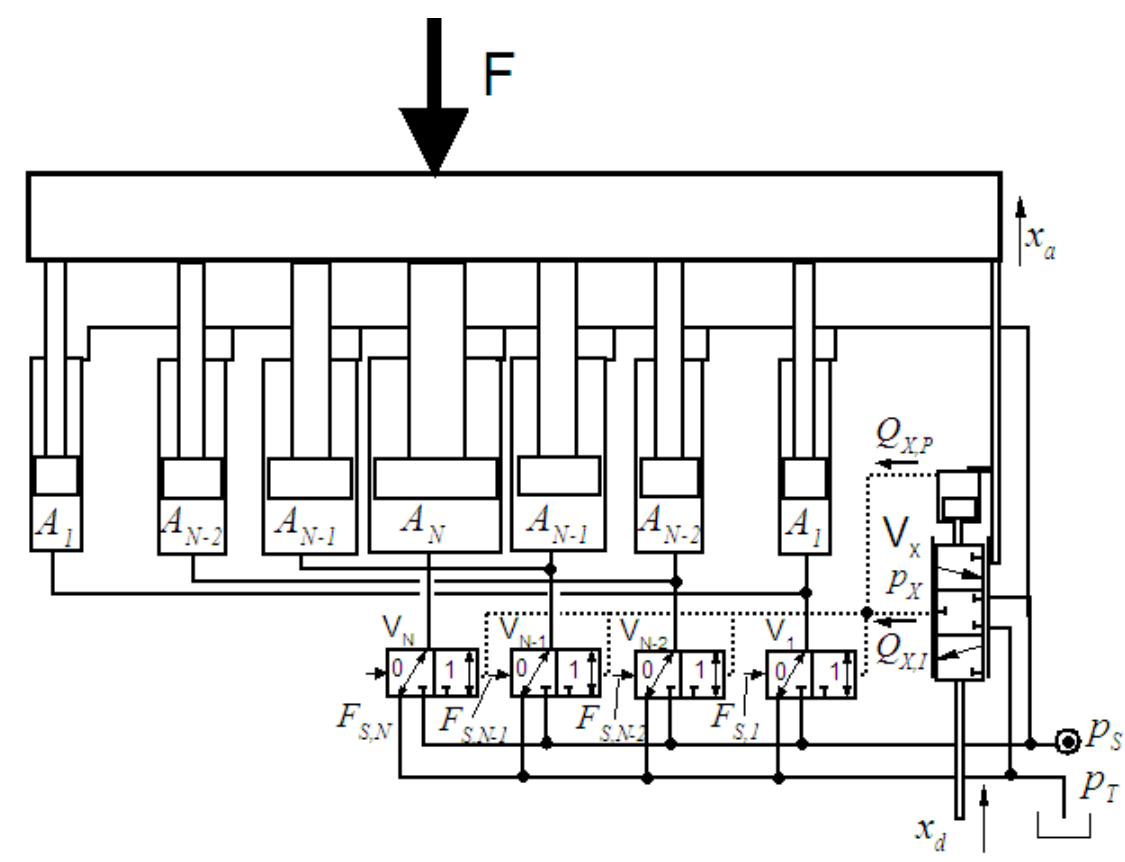

Figure 1: Linear digital hydraulic amplifier with a differential feed-back [1].
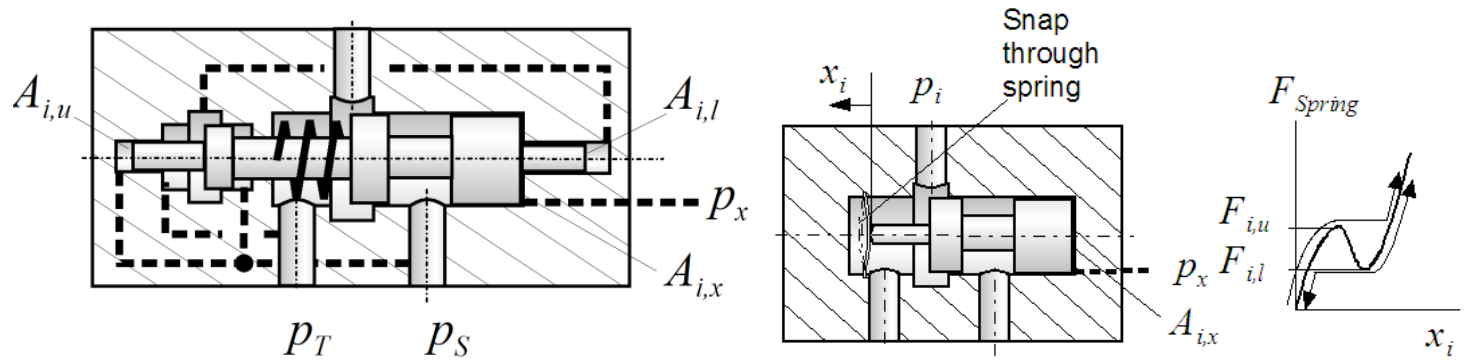

Figure 2: Exemplary embodiment designs of two concepts to realize a hysteresis behavior of a switching valve w.r.t. to a pilot pressure $p_{x}$; left: hydraulic feedback by additional metering edges; right: snap through spring.

In this paper the feedback from the pressure of the multi-chamber cylinders is studied instead. It saves extra features of the valves and is applicable to any valve size. The concept is presented and its basic functioning and behavior are analyzed by a simple mathematical model. An exemplary embodiment design is presented to demonstrate the practicability.

\section{Binary counting principle}

The binary counter principle is given by a schematic in Figure 3. For a better comprehension of the functioning Figure 5 and Figure 10 are helpful. The purpose of the circuitry is adjusting a hydraulic actuation force $F_{H}$ which 
is a four bit equivalent of the pressure $p_{C}$ in the pilot line. Thus, the counter can be also seen as a hydraulic analog-digital converter. For an equal force stepping the piston areas must follow a binary sequence. There are two solutions; both are given in (1); the first one is used in this paper.

$$
A_{1}: A_{2}: A_{3}: A_{4}=\left\{\left[-\frac{1}{8}: \frac{1}{4}:-\frac{1}{2}: 1\right],\left[-\frac{1}{8}: \frac{1}{2}:-\frac{1}{4}: 1\right]\right\}
$$

A negative area is one that causes a negative hydraulic force. This convention eases the formulation of several equations.

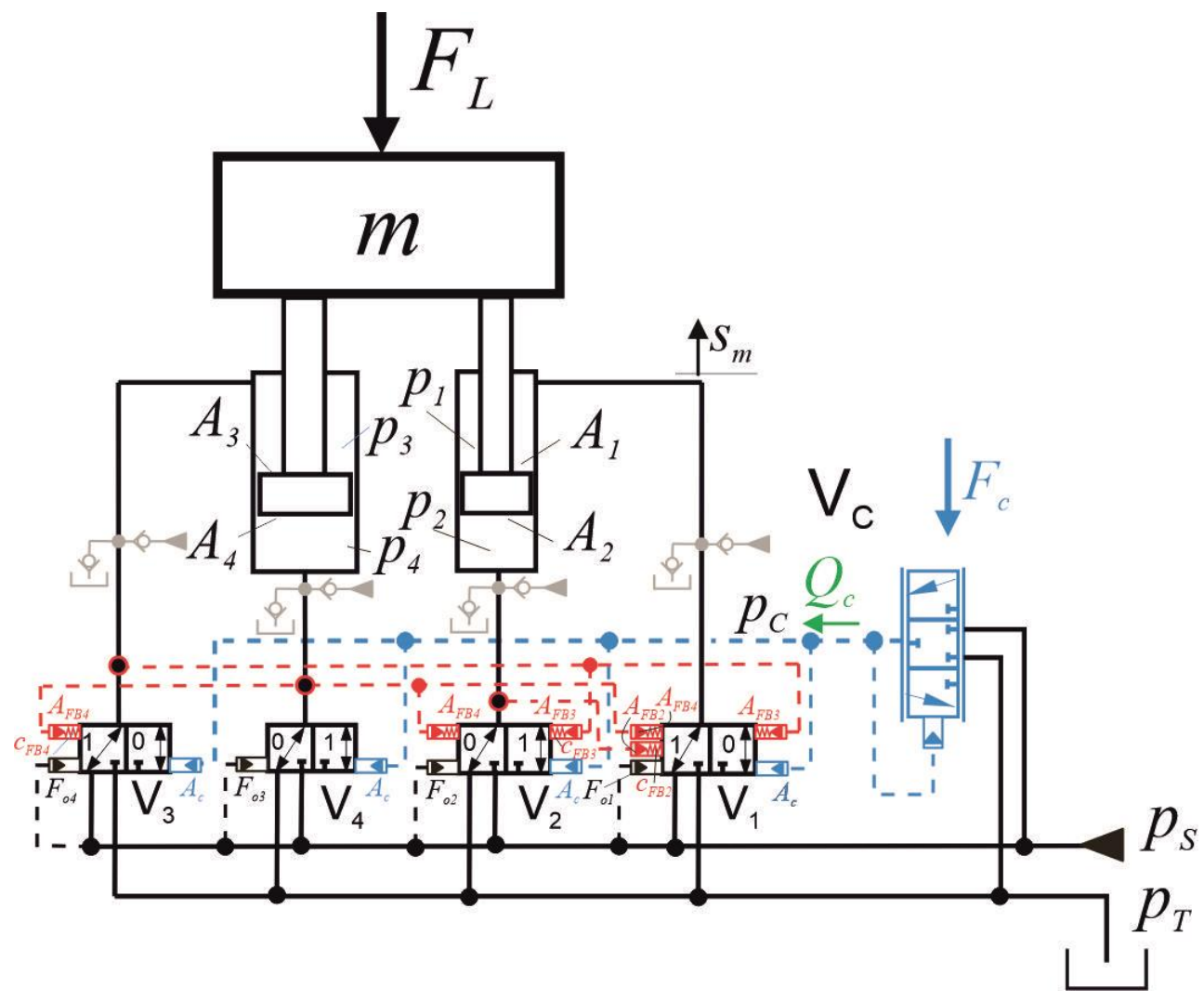

Figure 3: Hydraulic binary counting for the control of a four-chamber cylinder.

The force levels which the counter should realize and the corresponding switching positions of the four valve $\mathrm{V}_{1} . . \mathrm{V}_{4}$ are depicted in Figure 4. 


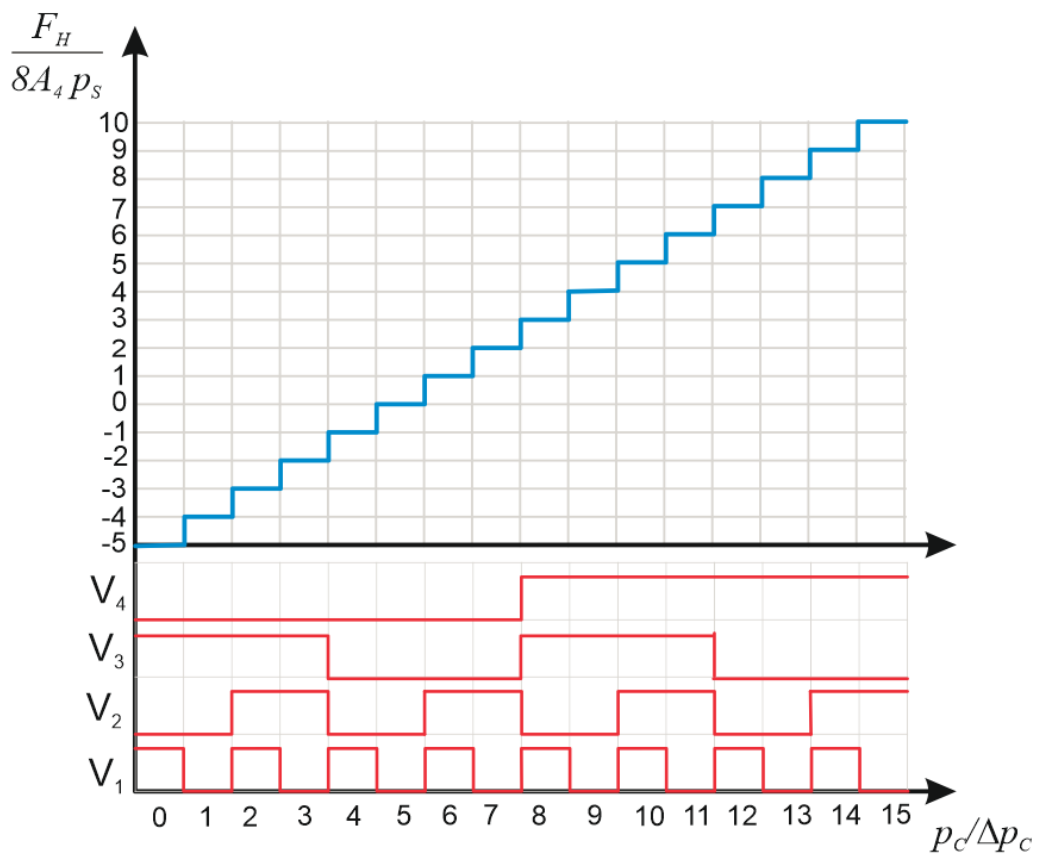

Figure 4: Intended AD conversion behavior of the binary counter and corresponding valve states.

If the pilot pressure is below the first threshold value $\left(p_{C}<\Delta p_{C}\right)$ all four valves are in their initial states, as sketched in Figure 3. $\Delta p_{C}$ are the pilot pressure increments, see Figure 4. The threshold values for the valves can be set hydraulically using the system pressure $p_{S}$ - as shown in Figure 3 - or by spring preload. In the first case, the magnitude of these preload forces $F_{O i, i=1 . .4}$ is adjusted by the active hydraulic displacement areas $A_{o i}, i=1 . .4$. The values of $F_{O i}$ are given in Table 1 .

Table 1: Values of the threshold forces $F_{O \mathrm{i}}$.

\begin{tabular}{|l|c|c|c|c|}
\hline Valve & $\mathrm{V}_{1}$ & $\mathrm{~V}_{2}$ & $\mathrm{~V}_{3}$ & $\mathrm{~V}_{4}$ \\
\hline$\frac{F_{O i}}{\Delta p_{C} A_{C}}$ & 5 & 6 & 4 & 8 \\
\hline$\frac{A_{F B k} p_{S}}{2 \Delta p_{C} A_{C}}$ & $\sim$ & 2 & 4 & 8 \\
\hline
\end{tabular}

The pressures in the cylinder chambers $p_{k}$ are fed back to the valve $\mathrm{V}_{\mathrm{i}}$ via areas $A_{F B k}$. Their sizes depend only on the chamber number $k$ and not on the valve number $i$. Thus, the corresponding piloting units of order $k$ can be realized in all valves identically. Valve $V_{i}$ needs feedback only from the higher order valves $i+1$ (2 .. 4). Valves $\mathrm{V}_{1}$ and $\mathrm{V}_{3}$ are controlling chambers with a hydraulic force in negative direction. Their initial values are 1 , hence, the pressures in the related chambers are $p_{S}$. First switching of $\mathrm{V}_{1}$ and $\mathrm{V}_{3}$ cause a pressure change from $p_{S}$ to $p_{T}$ which is opposite to that of valves $\mathrm{V}_{2}$ and $\mathrm{V}_{4}$. To handle this logic situation the feedback actuators of pressure $p_{3}$ are placed on the opposite sides. The corresponding initial forces are compensated by an increased value of $F_{O I}$ and $F_{O 2}$. This additional offset is $4 \triangle p_{C} A_{C}$. Without this the values of $F_{O 1}$ and $F_{O 2}$ would follow the binary steps 1 and 2 as is the case for $F_{O 3}$ and $F_{O 4}$ since valves $\mathrm{V}_{3}$ and $\mathrm{V}_{4}$ are not affected by $p_{3}$.

In order to keep the feedback forces in a narrow tolerance range even when the chamber pressure have some variation, a spring is placed between the feedback cylinders and the valve spools. The limited stroke of the feedback cylinder defines the feedback force via the spring constant. The areas $A_{F B k}$ are such that these springs are fully compressed with a fraction of the system pressure. For the values shown in this paper that fraction is taken to be $p_{K} / p_{S}=1 / 2$. This explains the number 2 in the denominator of the expression in the second row of Table 1.

When $p_{C}$ is raised and exceeds $\Delta p_{C} \mathrm{~V}_{1}$ starts to move from state 1 into state 0 . The transition is continuous first, due to the continuous force changes of the springs in the feedback units. The lower these spring constants $c_{F B k}$, the steeper the rise of the valve transition with rising $p_{C}$, but the higher the compression stroke of the feedback units. Valve $\mathrm{V}_{2}$ starts switching if $p_{C}>2 \Delta p_{C}$. When it reaches state 1 , the pressure $p_{2}$ rises and puts a feedback 
force $F_{O 2}$ on valve 1 which makes it switching back when it reaches $p_{S} / 2$. At $p_{C}=3 \Delta p_{C}$ switching of $\mathrm{V}_{1}$ starts again. At $p_{C}=4 \Delta p_{C} \mathrm{~V}_{3}$ goes from state 1 to 0 which finally causes $\mathrm{V}_{1}$ and $\mathrm{V}_{2}$ to switch back to their initial states 1 and 0 , respectively. Valve $\mathrm{V}_{4}$ begins its transition to state 1 when $p_{C}=8 \Delta p_{C}$. Counting down, i.e. lowering the output force, happens in an analog way.

As will be demonstrated in Section 4 by simulation results, a monotonous rise of $p_{C}$ does not mean a continuously positive pilot flow rate $Q_{C}$. For instance, if $\mathrm{V}_{4}$ is switched the lower order valves $\mathrm{V}_{1.3}$ switch back and displace three times the fluid which $\mathrm{V}_{4}$ takes back to the pressure control valve $\mathrm{V}_{\mathrm{C}}$. Thus, in sum that event leads to a negative flow. That circumstance requires a control of $p_{C}$ as indicated in the schematic of Figure 3 . It shows as actual input a force $F_{C}$, which can be a manual or magnetic force. In the latter case $\mathrm{V}_{\mathrm{C}}$ represents a proportional three way pressure control valve. Basically, it would be also possible to avoid flow reversal when a higher order valve switches, if the displacement volume of the pilot pressure actuation cylinder of $\mathrm{V}_{\mathrm{i}}$ is larger than that of all lower order valves. That means an exponential growth of these displacement volumes whereas in the concept proposed here all valves $\mathrm{V}_{1 . .4}$ differ only with respect to $F_{O i}$ or $A_{O i}$, respectively.

The metering edges geometry (overlap or underlap) of the sealing lands decide on the transition between the two topologic different situations; to have an intermediate short circuiting from pressure to tank line or to happen fully separately. To avoid an intermediate blocking of the cylinder chambers or cavitation in the latter case check valves can be placed to both supply lines. These optional valves limit the pressures to the range $\left[p_{T}, p_{S}\right]$ and are shown in grey color in Figure 3. The problem of system transients due to switching and measures to avoid or limit unwanted dynamical effects are not studied in this paper.

\section{Mathematical model of the counter}

The validity of the concept and the influence of the design parameters on the properties are investigated by a simple mathematical model. It considers only effects relevant for the basic working principle and comprises the four valves and the pressure build-up in the four cylinder chambers. The following effects are disregarded:

(1) inertias of the spools and pistons of the valve piloting pistons,

(2) flow and friction forces in valves $V_{i}$,

(3) influence of motion of the driven system $\left(\dot{s}_{m}\right)$ for the pressure build-up dynamics in the cylinder chambers.

Simplifications (1) and (3) are based on the assumption that there is a clear order of the dynamics of the motion of the driven system $s_{m}(t)$, the pressure build up dynamics in the cylinder $p_{k}(t)$, and the valve response dynamics $s_{V i}(t)$, with sufficient frequency gaps in between. Assumption (2) represents a requirement on valve design and dimensioning, particularly of the control force steps $\Delta p_{C} A_{C}$. This force increment must dominate all parasitic effects neglected according to this assumption.

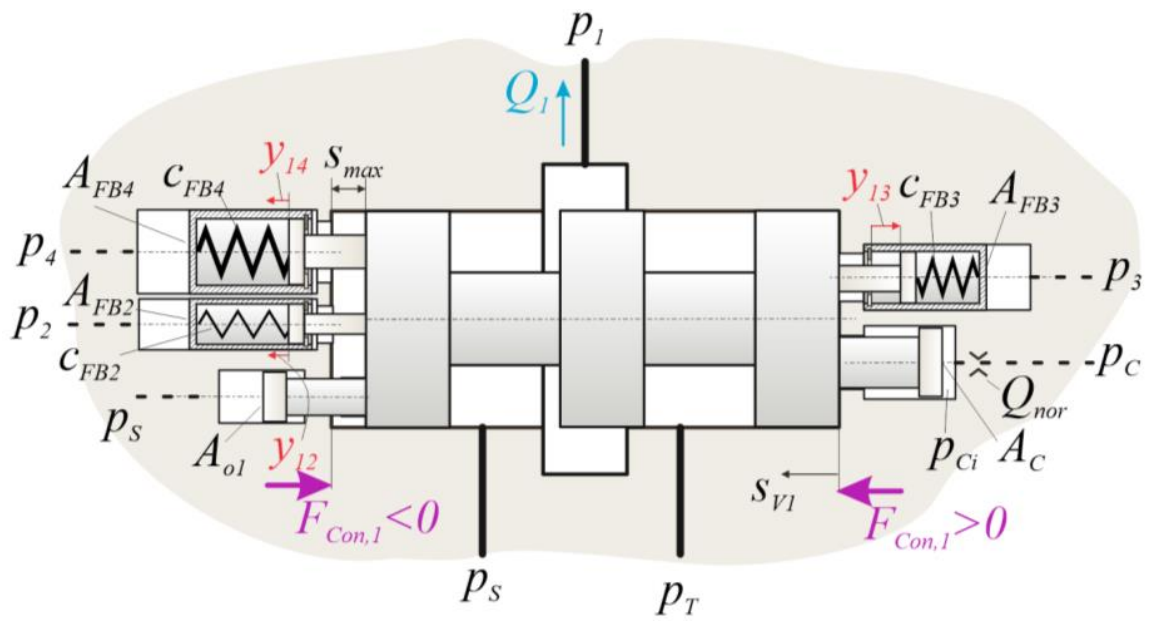

Figure 5: Model of valve $V_{1}$; other valves have lower number of feed-back actuators and different values $A_{\text {oi }}$.

The most complex valve is $\mathrm{V}_{1}$. A sketch of the main functional components is given by Figure 5 . The spool has a maximum travel distance $s_{\max }$ which is identical for all valves. A perfect metering of the 3-2 way valves is assumed, the relation between spool travel $s_{V i}$ and nondimensional valve position $y_{i}$ is 


$$
y_{i}=-1+2 \frac{s_{V i}}{s_{\max }},
$$

and for the flow rate $Q_{i}$ over the valve $\mathrm{V}_{\mathrm{i}}$ to the related cylinder chamber

$$
\begin{aligned}
& Q_{i}=Q_{N}\left[\operatorname{sg}\left(y_{i} \operatorname{sign}\left(A_{i}\right)\right) \hat{\sqrt{\frac{p_{S}-p_{i}}{p_{N}}}}-\operatorname{sg}\left(-y_{i} \operatorname{sign}\left(A_{i}\right)\right) \sqrt{\frac{p_{i}-p_{T}}{p_{N}}}\right] . \\
& \operatorname{sg}(x)=\max (0, \min (x, 1)) ; \sqrt[\wedge]{x}=\sqrt{|x|} \operatorname{sign}(x)
\end{aligned}
$$

The equilibrium of the up to five actuation forces for the spool valve $V_{i}$ are

$$
p_{C i} A_{C}-F_{O i}+F_{C o n, i}-\sum_{k=i+1}^{4} F_{F B k i} \operatorname{sign}\left(A_{k}\right)=0 .
$$

The factor $\operatorname{sign}\left(A_{k}\right)$ considers the feedback actuators' individual force direction. The forces are related to pressures and positions by the following rules.

$$
\begin{aligned}
& F_{O i}=A_{O i} p_{S} ; \quad F_{F B k i}=\min \left[p_{k} A_{F B k},\left(y_{i k}+y_{k 0}\right) c_{F B k}\right] \operatorname{sign}\left(A_{k}\right) \\
& y_{i k}=0 \\
& y_{i k}=\left\{\begin{array}{cc}
s_{V i} & \text { for } k=2,4 p_{k} A_{F B k}<y_{k 0} c_{F B k} \\
s_{\max }-s_{V i} & \text { for } k=3
\end{array} \text { if } p_{k} A_{F B k} \geq y_{k 0} c_{F B k}\right. \\
& F_{C o n, i} \geq 0 \text { if } s_{V i}=0 \\
& F_{C o n, i} \leq 0 \text { if } s_{V i}=s_{\max } \\
& s_{V i} \in\left[0, s_{\max }\right]
\end{aligned}
$$

The contact force $F_{c o n, i}$ assures equilibrium if the spool is in one of its end positions $s_{V i}=\left\{0, s_{\max }\right\}$. The pressure build-up equation for chamber $i$ reads:

$$
\dot{p}_{i}=\frac{E}{V_{i 0}+A_{i} s_{m}}\left(Q_{i}-\dot{s}_{m} A_{i}\right)
$$

The pilot flow $Q_{C}$ and its integral $V_{Q C}$ are related to the control valve $\left(\mathrm{V}_{C}\right)$ equation.

$$
\begin{aligned}
& \dot{V}_{Q C}=Q_{C}=s g\left(\frac{y_{C}}{y_{C \max }}\right) Q_{N C} \sqrt{\frac{p_{S}-p_{C}}{p_{N}}}-s g\left(-\frac{y_{C}}{y_{C \max }}\right) Q_{N C} \hat{\sqrt{\frac{p_{C}-p_{T}}{p_{N}}}} \\
& y_{C}=\frac{F_{C}-p_{C} A_{C C}}{c_{C C}}
\end{aligned}
$$

The pressure in the pilot line $p_{C}$ is related to the difference of the inflowing oil volume and displacement by the valves $\mathrm{V}_{\mathrm{i}}$ due to fluid compressibility in the pilot system, given by the hydraulic capacitance $C_{H p}$.

$$
p_{C}=\frac{1}{C_{H p}}\left(V_{Q C}-A_{C} \sum_{i=1}^{4} s_{V i}\right)
$$

The speeds of the spool motions are related to the pressures $p_{C I .4}$ in the displacement chambers of the pilot system by the following equation.

$$
\dot{\mathrm{s}}_{V i}=\frac{Q_{N o r}}{A_{C}} \sqrt{\frac{p_{C}-p_{C i}}{p_{N}}} ; \mathrm{i}=1 . .4
$$

The orifice between pilot line and the displacement chambers (specified by a nominal flow rate $Q_{N o r}$ ) are inserted to ease simulation. Otherwise $p_{C}$ and $s_{V i}$ are not directly related to each other but only by (4) and (8) which involve the strongly nonlinear contact problems due to the contact forces $F_{C o n, i}$. The solution of this set of equations requires methods to solve multidimensional contact problems. 
The behavior of the system is studied for a ramp up and down of control input $F_{C}$. According to the simplifications specified above, the process is considered being so fast that no substantial change of the position $s_{m}$ takes place in that time. Furthermore, the valves nominal flow rates $Q_{N}$ are considered being large enough to keep the pressure losses small compared to system pressure $p_{S}$, a condition to cancel the term $\dot{s}_{m}$ in (6). The reason for the simplifications is mainly to keep the analysis general and to keep the number of parameters which influence the results small. Then the whole system has the following state vector $\mathbf{x}$.

$$
\mathbf{x}=\left[p_{1 . .4}, V_{Q C}, s_{V 1 . .4}\right]^{T}
$$

It contains nonlinear constraint relations due to the contact forces $F_{C o n, i}$ in (4). In order to keep the simulation model simple, these forces are modeled as elastic contact forces according to the following rule.

$$
F_{C o n, i}=c_{C o n}\left(s g 1\left(-s_{V i}\right)-s g 1\left(s_{V i}-s_{\max }\right)\right) ; \operatorname{sg} 1(x)=\max (0, x)
$$

The vector describing the flow of $\mathbf{x}$ is given by equations (6), (7) and (9) in combination with equations (4), (5), (8) to compute $p_{C i}$ and $p_{C}$. The model is implemented in a Matlab program. The differential equations system is stiff, therefore, solutions are computed numerically using Matlab's stiff ODE solver ode15s.

\section{Simulation results}

The counter's behavior is studied for an exemplary four chamber cylinder drive for exoskeleton use. The used system parameter values set are listed in Table 2.

Results are given by plots in the next figures. They show the working of the system. The response dynamics is illustrated for the switching from stage 3 to 4 , when 3 valves change position, by the diagram in Figure 8 . Despite the small nominal flow rate of the pressure control valve (see $Q_{N C}$ in Table 2) valve $\mathrm{V}_{3}$ needs only 3 milliseconds for switching. The lower order valves $V_{1 . .2}$ need much less due to the strong effect of the feedback mechanism.

The small changes of valve position at fully open or close positions, best to be seen for $s_{V 4}$, results from the modeling of the elastic end stop with the spring constant $c_{C o n}$ and the effect of $p_{C}$. This is a modeling issue and has no meaning for the functioning of the counter.

Table 2: System and Matlab 'ode15s'options parameter values for simulation study.

\begin{tabular}{|l|l|l|l|l|l|}
\hline Parameter & Value & Parameter & Value & Parameter & Value \\
\hline$A_{C}$ & $10 \mathrm{~mm}^{2}$ & $A_{C C}$ & $2 \mathrm{~mm}^{2}$ & $A_{F B 2.3}$ & {$[1.63 .26 .4] \mathrm{mm}^{2}$} \\
\hline$A_{k k=1 . .4}$ & {$[-.125 .25-0.51] \mathrm{cm}^{2}$} & $c_{F B 1.3}$ & {$[0.81 .63 .2] \mathrm{N} / \mathrm{mm}$} & $c_{C C}$ & $5.33 \mathrm{~N} / \mathrm{mm}$ \\
\hline$c_{C o n}$ & $1000 \mathrm{~N} / \mathrm{mm}$ & $C_{H p}$ & $0.0643 \mathrm{~mm}^{3} / \mathrm{bar}$ & $E$ & $14000 \mathrm{bar}$ \\
\hline$y_{k 0 k=1 . .4}$ & {$[10101010] \mathrm{mm}$} & $y_{C m a x}$ & $0.3 \mathrm{~mm}$ & $F_{o i ~ i=1 . .4}$ & {$[20241632] \mathrm{N}$} \\
\hline$\Delta p_{C}$ & $4 \mathrm{bar}$ & $p_{N}$ & $100 \mathrm{bar}$ & $p_{S}$ & $5 \mathrm{bar}$ \\
\hline$p_{T}$ & $0 \mathrm{bar}$ & $Q_{N C}$ & 1 lit/min & $Q_{N}$ & 3 lit/min \\
\hline$Q_{N o r}$ & $0.5 \mathrm{lit} / \mathrm{min}$ & $s_{m}$ & $50 \mathrm{~mm}$ & $s_{\max }$ & $0.5 \mathrm{~mm}$ \\
\hline$V_{i 0 \text { i }=1.4}$ & {$[1.50 .56 .02 .0] \mathrm{cm}^{3}$} & \multicolumn{2}{|l|}{ options $=$ odeset('RelTol',1e-4,'AbsTol',1e-8) } \\
\hline
\end{tabular}



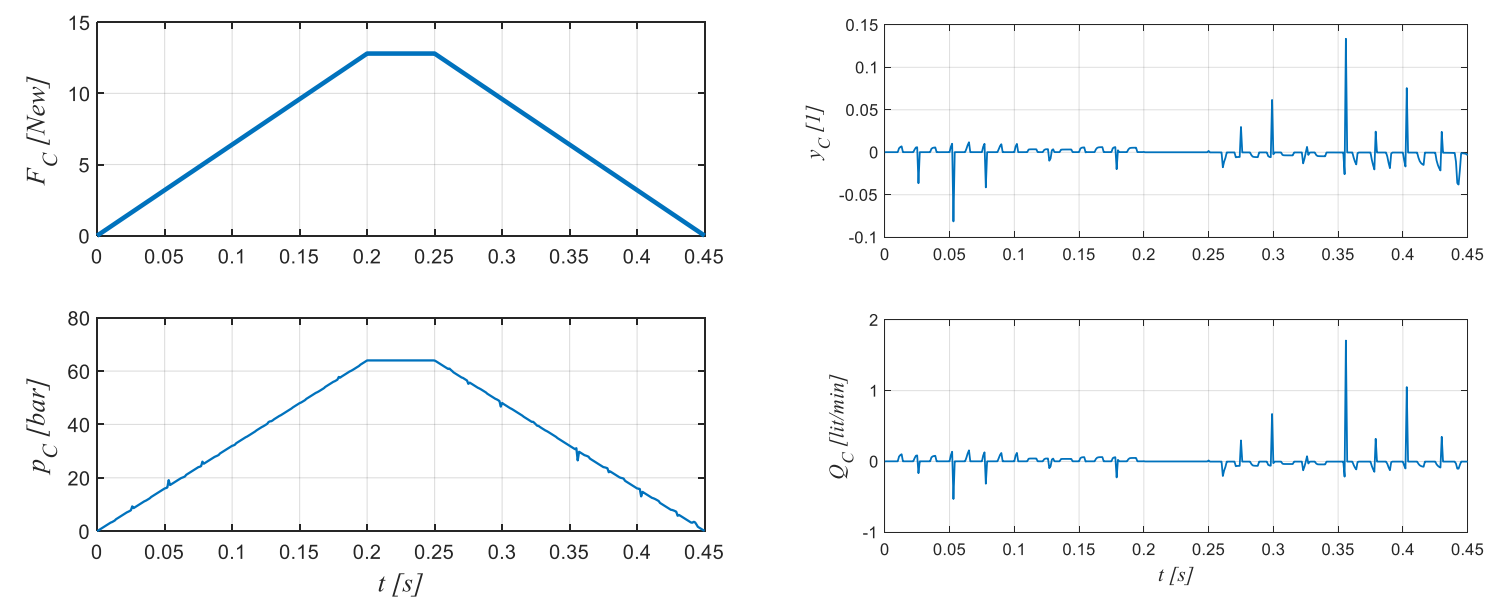

Figure 6: Results of a simulation of the digital cylinder drive controlled by a binary counter system; input force $F_{C}$, pilot pressure $p_{C}$, pressure control valve nondimensional opening $y_{C}$, and pilot flow $Q_{C}$.
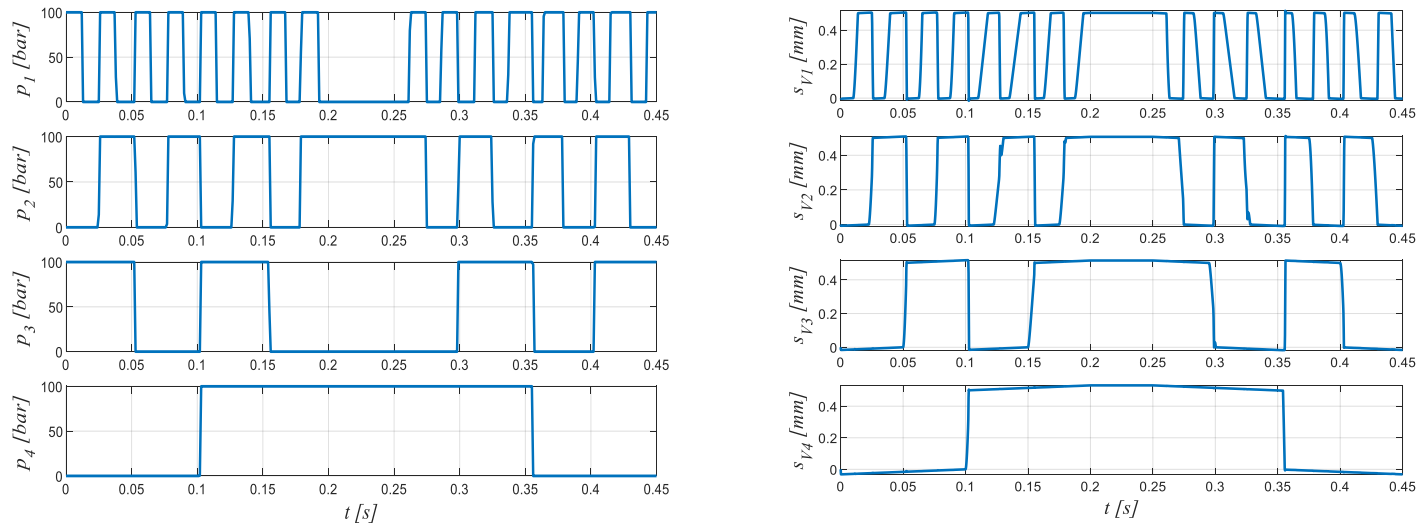

Figure 7: Results of a simulation of the digital cylinder drive controlled by a binary counter system; pressures in cylinder chamber $p_{i}$ and valve positions $s_{V i}$.

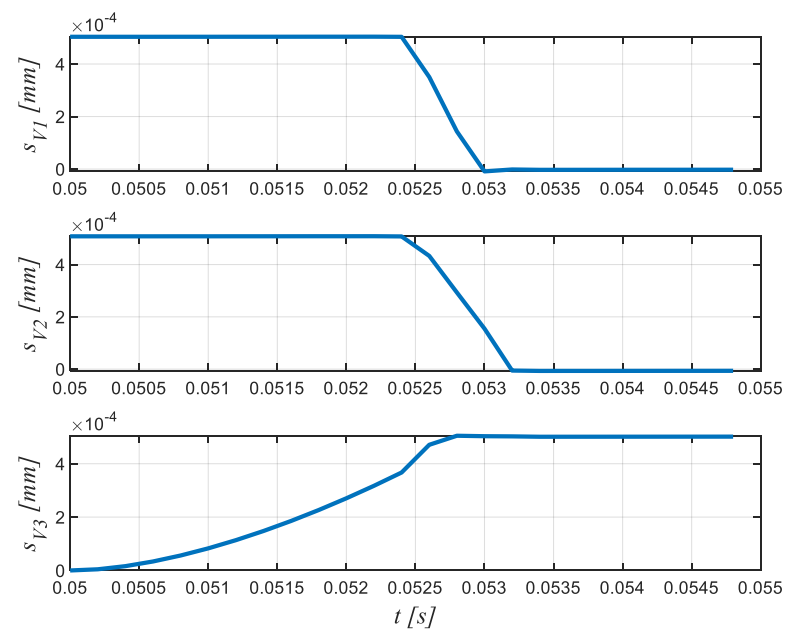

Figure 8: Magnified view on the response diagrams of valves $V_{1.3}$ when the counter switches from stage 3 to 4 .

The system with the parameters of Table 2 can also follow faster changes of the system input $F_{C}$. Figure 9 shows the response in terms of valve position and chamber pressures for a ramp on and off time of 50 milliseconds. The system is probably at its limits when counting down, even though the cylinder pressure $p_{l}$ follows the counting sequence better than the spool of valve $\mathrm{V}_{1}$. 

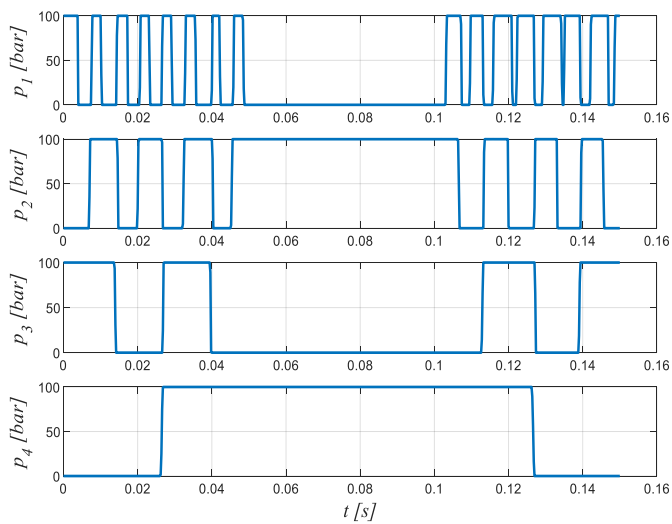
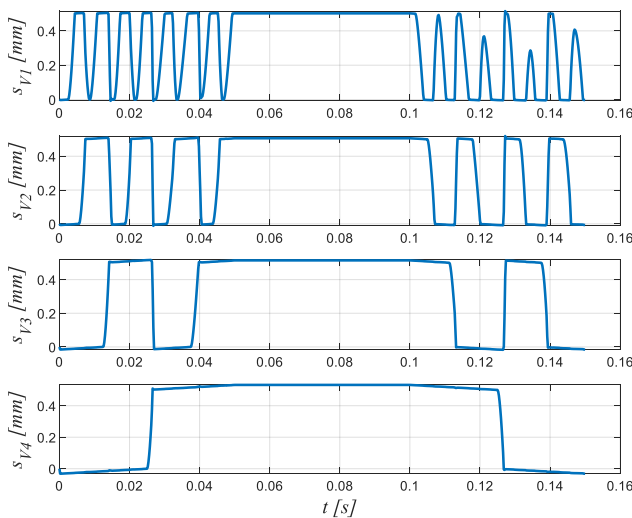

Figure 9: Results of a simulation of the digital cylinder drive controlled by a binary counter system for a ramp time of 50 milliseconds; pressures in cylinder chamber $p_{i}$ and valve positions $s_{V i}$.

\section{Valve design}
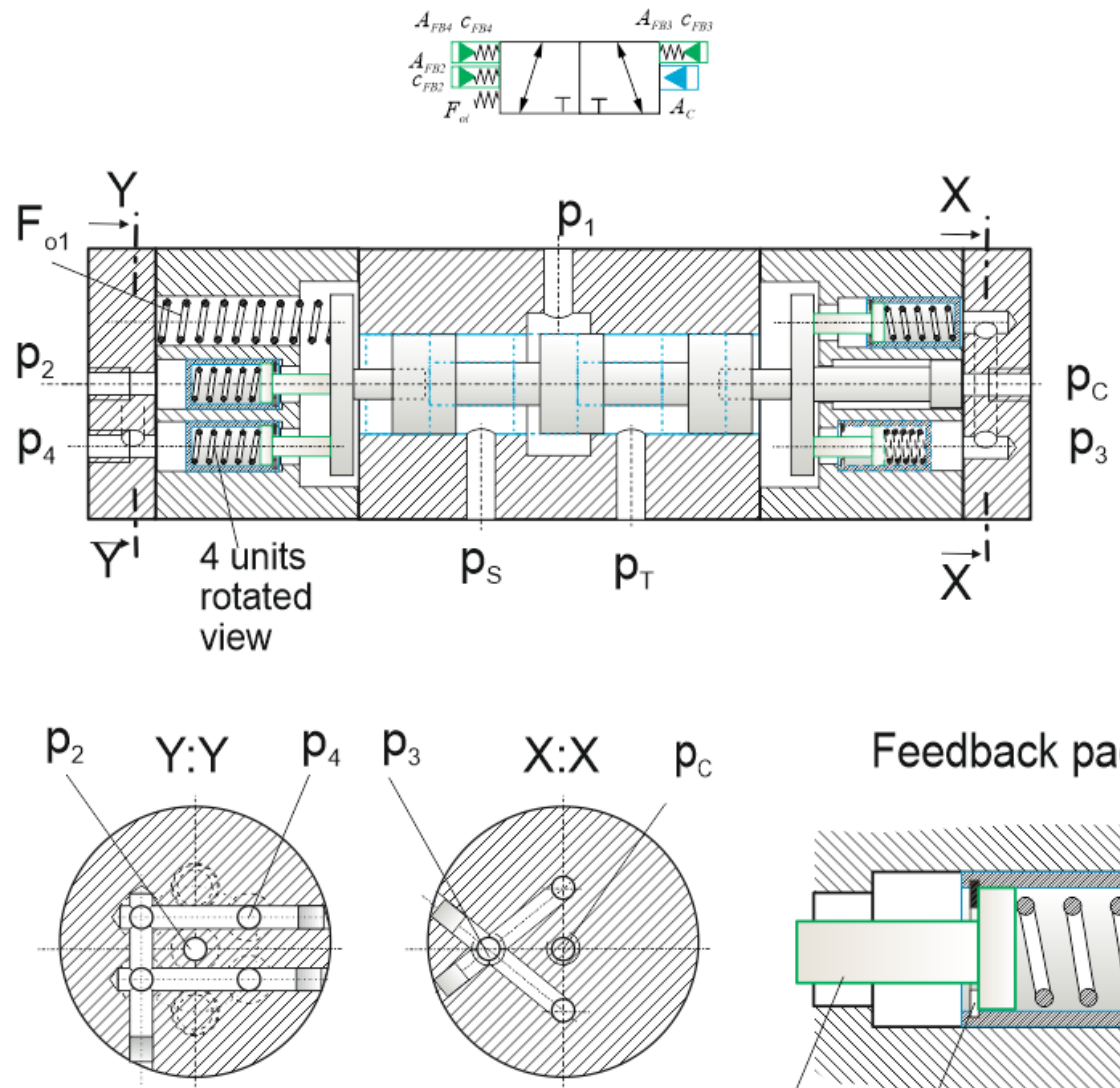

Feedback package

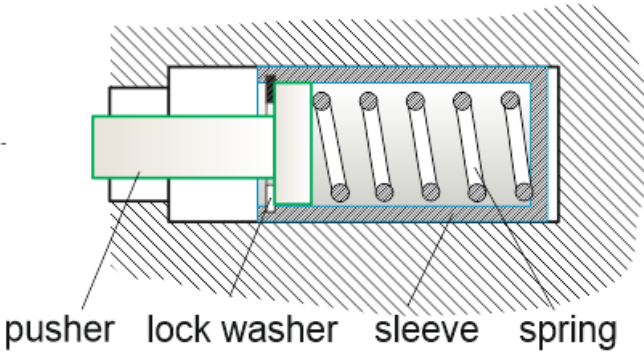

Figure 10: Exemplary design of valve $V_{l}$.

The valve consists of a standard 3-2 way valve w.r.t. the main stage but needs an elaborate piloting system with up to five separate inputs. To judge on the feasibility of a practical realization an exemplary embodiment design is presented in this section.

Figure 10 shows such a design of valve $\mathrm{V}_{1}$ which is the most complex one. Higher stage valves can be widely identical. The piston areas $A_{C}$ and all cylinder pressure feedback units are identical for all valves. Higher order valves need fewer number of feedback units, $\mathrm{V}_{2}$ from $p_{3}$ and $p_{4}, \mathrm{~V}_{3}$ only from $p_{4}$, and $\mathrm{V}_{4}$ none. All valves could be equipped with all feedback units and only the required ones are actually connected to the cylinder chambers or unneeded units are skipped. The feedback units of each valve are only made of one feedback package, consisting of bore, sleeve, lock washer and pusher. The higher forces of the higher order feedback units - they 
follow in value the power series of 2 - are realized by a higher number of feedback packages. $p_{2}$ has one unit, $p_{3}$ two units, and $p_{4}$ four units.

For a fast response of a valve when the pilot pressure reaches the corresponding value, the springs in these units should have a relatively large pretension. In the simulations a pretension $y_{k 0} k=1 . .4$ of $10 \mathrm{~mm}$ was set which is twenty times the spool stroke $s_{\max }=0.5 \mathrm{~mm}$. In order to save space the concept with the sleeves which holds the pre-tensioned spring with the lock washer is recommended. Without this pretension sleeve the spring must be given the full pretension stroke to avoid a force on the spool when the related feedback pressure is zero. That increases the valve size unnecessarily. Furthermore, that would cost more fluid from the cylinder chamber when the pressure switches from tank to system pressure. The only valve specific parameter is the threshold force $F_{o i}$ $i=1.4$. In the design concept of Figure 10 it is realized via a spring, actually two springs in order to balance that force with respect to the centerline. The simplest way to realize the different force values ([20 2416 32]) is by different pretensions $\left(y_{F o i}\right)$ of relatively soft springs to guarantee $s_{\max }>y_{F o i}$. Individual pretensions are best realized by different washers placed between spring and end cap. This washer is not shown in Figure 10.

\section{Summary and conclusion}

A concept to control a binary staggered four chamber cylinder system by a hydraulic binary counter was presented. It uses feedback of the pressures from the higher order chambers to accomplish the reset of the lower order valves if a binary jump of a certain order is to be made. That concept needs piloted 3-2 way valves and a pilot pressure control system, which transfers the desired force level into a corresponding pilot pressure. The counter transfers this pressure into an appropriate force level out of the sixteen possible force levels available of a four chamber cylinder system. The functioning of this concept was demonstrated by a numerical simulation of a simple system model. A small hydraulic actuator for an exoskeleton served as an example.

The paper does not yet present a study of the control performance of certain motion control tasks, for instance, of exoskeletons. This and a compact realization of the pilot pressure control valve will be tackled next.

The concept can be applied to larger hydraulic drives as well, for instance for the four chamber cylinders for excavators, brought into discussion in the last years. In this case, the system needs to be augmented by means for a continuous force control for certain operation modes, obviously by adding some throttling control. A hydromechanical concept which can do both, combined digital and throttling control when inevitable, and pure digital control whenever feasible, will be a topic of further research.

\section{Nomenclature}

\begin{tabular}{ll}
\hline Designation & Denotation \\
\hline$A_{F B k k=2 . .4}$ & Piston areas for pressure feedback to valve $\mathrm{V}_{\mathrm{i}}$ by pressure $p_{k}$ \\
$A_{C}$ & Pilot piston area \\
$A_{C C}$ & Pilot pressure feedback area of pressure control valve $\mathrm{V}_{\mathrm{C}}$ \\
$A_{k k=1 . .4}$ & Four chamber piston areas \\
$A_{O i \text { i } 1 . .4}$ & Piston areas for hydraulic realization of offset forces $F_{O i}$ \\
$C_{H p}$ & Hydraulic capacitance of pilot line \\
$E$ & Compression modulus \\
$F_{F B k i=1 . .3, k=i+1 . .4}$ & Feedback forces to spool of valve $\mathrm{V}_{\mathrm{i}}$ from pressure $p_{i}$ \\
$F_{C}$ & Control input force to pressure control valve $\mathrm{V}_{\mathrm{C}}$ \\
$F_{C o n, i=1 . .4}$ & Contact force to spool at end stop positions \\
$F_{H}$ & Hydraulic force of the four chamber cylinder \\
$F_{L}$ & Load force \\
$F_{O i \text { i }}$ & Force to realize switching threshold value for pilot pressure \\
$Q_{C}$ & Flow rate from pressure control valve into pilot system \\
$Q_{N}$ & Nominal flow rate of valve main stages \\
$Q_{N C}$ & Nominal flow rate of pressure control valve \\
&
\end{tabular}




\begin{tabular}{|c|c|}
\hline$Q_{\text {Nor }}$ & Nominal flow rate of orifice \\
\hline$V_{i 0} i=1 . .4$ & Dead volumes of cylinder chambers for $s_{m}=0$ \\
\hline$V_{Q C}$ & Displacement volume of $Q_{C}$ \\
\hline$c_{C C}$ & Spring constant of pressure control valve \\
\hline$c_{\text {Con }}$ & Spring constant of contact force modeling \\
\hline$c_{F B k k=1 . .3}$ & Spring constant of pressure feedback units \\
\hline$i, k$ & Indices of valve and cylinder chamber numbers \\
\hline$m$ & Load inertia \\
\hline$p_{C}, \Delta p_{C}$ & Pilot pressure and its counting increment \\
\hline$p_{C i i=1 . .4}$ & Pressure acting on pilot area $A_{C}$ of at valve $\mathrm{V}_{\mathrm{i}}$ \\
\hline$p_{k k=1 . .4}$ & Pressure in cylinder chamber $k$ \\
\hline$p_{N}$ & Nominal pressure loss of valves or orifices \\
\hline$p_{S}, p_{T}$ & System and tank pressure \\
\hline$s_{m}$ & Cylinder travel \\
\hline$s_{\max }$ & Spool stroke of valves \\
\hline$S_{V i i=1 . .4}$ & Position of spool of valve $V_{i}$ \\
\hline $\mathbf{x}$ & State vector of dynamical model \\
\hline$y_{c}$ & Position of pressure control valve \\
\hline$y_{\text {cmax }}$ & Stroke of pressure control valve \\
\hline$y_{i k}$ & Compression of feedback spring by pressure $p_{K}$ in valve $V_{i}$ \\
\hline$y_{k 0}$ & Pre-compression of feedback spring \\
\hline
\end{tabular}

\section{Acknowledgement}

This works was done in the framework of the COMET K2 Center on Symbiotic Mechatronics, which is funded by the Austrian Federal Government, the State Upper Austria and by its Scientific and Industrial Partners.

\section{References}

[1] Biedermann I., Scheidl R., Plöckinger A. (2011). A Linear Digital Hydraulic Amplifier, in: Proceedings of the Fourth Workshop on Digital Fluid Power, 21-22 September, 2011, Linz, Austria, pp. 75-89.

[2] Scheidl, R., \& Mittlböck, S. (2018). A hydraulic piloting concept of a digital cylinder drive for exoskeletons. In BATH/ASME 2018 Symposium on Fluid Power and Motion Control. American Society of Mechanical Engineers Digital Collection.

[3] Scheidl, R., \& Mittlböck, S. (2018). A mathematical analysis of a hydraulic binary counter for hydraulic exoskeleton actuation. International Journal of Hydromechatronics, 1(2), 153-171.

[4] Matthias Scherrer, Simon Mittlböck, Rudolf Scheidl, "Embodiment Design of a Hydraulic Binary Counter for Exoskeleton Use - Problems and New Solutions" : Proceedings of the 10th Workshop on Digital Fluid Power (DFP19), February 28th-March 1st 2019, Linz, Austria, 3-2019.

[5] Matthias Scherrer, Erwin Hauser, Rudolf Scheidl, "Simulation study of permanent magnetic actuation for a hydraulic valve with hysteresis response behavior" : Proceedings of the 2020 ASME on Fluid Power and Motion Control FPMC2020 September 9-11, 2020, University of Bath, Bath, United Kingdom, 9-2020. 
[6] Linjama, M., Vihtanen, H. P., Sipola, A., Vilenius, M. (2009). Secondary controlled multi-chamber hydraulic cylinder. In The 11th Scandinavian International Conference on Fluid Power, SICFP (Vol. 9, pp. 2-4).

[7] Norrhydro (2018). Amazing energy saving for hydraulic machines, https://www.youtube.com/watch?v=qEUYtT-CWuo, visited May, 12, 2018.

[8] Dell'Amico A, Carlsson M., Norlin E., Sethson M. (2013). Investigation of a Digital Hydraulic Actuation System on an Excavator Arm. In13th Scandinavian International Conference on Fluid Power; June 3-5; 2013; Linköping, Sweden, pp. 505-511.

[9] Heybroek K. and Sahlman M. (2018) A hydraulic hybrid excavator based on multi-chamber cylinders and secondary control - design and experimental validation, International Journal of Fluid Power, DOI: $10.1080 / 14399776.2018 .1447065$ 